\title{
Isolation of urinary micro-vesicles from urine containing abundant proteins
}

\begin{abstract}
Recent studies have shown that urinary micro-vesicles (MVs) constitute a novel cellular source of biomarkers for kidney diseases. Although the available ultracentrifugation and ultrafiltration protocols effectively isolate urinary MVs, these procedures are ineffective for MVs isolation from urine containing abundant soluble proteins as found in different kidney diseases. Hence, we present a simple and convenient gel sieving chromatography based method that improves the isolation of MVs from urine containing abundant soluble proteins. Spot urine was collected from type- 1 diabetes mellitus patients (albumin to creatinine ratio, $\mathrm{ACR}>300 \mathrm{mg} / \mathrm{g}$, proteins $=4+$ ) and nonproteinuria healthy subjects $(\mathrm{ACR}<30 \mathrm{mg} / \mathrm{g}$, proteins $=$ nil). MVs were concentrated using ultracentrifugation followed by gel exclusion column chromatography. Urinary MVs fractions were collected and checked on Western blot using exosome-specific antibodies. The results showed significant removal of soluble proteins from MVs of proteinuria. Hence, this technique represents an easy and cost effective approach that could be efficiently utilized for partial purification of MVs from urine containing high proteins.
\end{abstract}

Keywords: exosomes, wilms' stumor, tsg101
Volume 2 Issue I - 2015

\author{
Anuradha K,',2 Pankaj C,' Munesh K, ${ }^{2}$ Eesh \\ $B,{ }^{2}$ Swasti $T^{2}$ \\ 'Department of Physiology and Biophysics, University of \\ Louisville, USA \\ ${ }^{2}$ Department of Molecular Medicine and Biotechnology, Sanjay \\ Gandhi Post Graduate Institute of Medical Sciences, India
} Correspondence: Anuradha Kalani, Department of Physiology
and Biophysics, University of Louisville, Louisville, KY, USA, Tel I5022027I79, Email anukalani@gmail.com

Received: December 08, 2014 | Published: January 14, 2015
Abbreviations: MVs, micro-vesicles; THP, tomm-horsfall protein; DTT, dithiothreitol

\section{Introduction}

Micro-vesicles (exosomes and microparticles) are released by renal tubules in the urine and constitute rich source of renal biomarkers for example: Wilm's tumor-1 (WT)-1, podocalyxin, $\alpha_{1}$-antitrypsin, aminopeptidase $\mathrm{N}$, vasorin precursor, ceruloplasmin..$^{1,2}$ Exosomes $(30-100 \mathrm{~nm})$ are formed by merocrine synthesis (inward budding and exocytosis) while microparticles $(100-1000 \mathrm{~nm})$ are formed by apocrine synthesis (surface shedding). Normal urine micro-vesicles (MVs) can be concentrated by ultracentrifugation and ultrafiltration ${ }^{3,4}$ but these procedures are ineffective in case of high proteinuricurine (protein $>4+$ ) as large number of other soluble proteins are retained with MVs. ${ }^{5}$ These abundant proteins mask the markers of interest. ${ }^{5}$ We examined two MVs preparations, one from type 1 diabetic patient's urine containing highly abundant soluble proteins and one from normal urine. Characteristics of the urine from the diabetic patient consisted of; specific gravity $=1.015, \mathrm{pH}=7.5$, Proteins $=4+$, Erythrocyte $=2+$, ACR $>300 \mathrm{mg} / \mathrm{g}$. While normal urine characteristics were; specific gravity $=1.005, \mathrm{pH}=7$, Proteins $=$ nil, Erythrocytes $=$ nil, $\mathrm{ACR}=8$. MVs where prepared as previously described using the standard ultracentrifugation methods. ${ }^{3}$ Type-1 diabetic MVs and normal urine MVs preparations were examined via SDS-PAGE and immuno-recognized with MVs specific anti-tumor susceptibility gene 101 (TSG101) 2,6 antibody using Western blot. A large background of unwanted soluble proteins was observed on Western blot with macroproteinuria MVs as compared to MVs isolated from normal urine (Figure 1). These abundant unwanted soluble proteins retain with MVs proteins and create problems in biomarker search during kidney diseases leading to high proteinuria urine. In order to purify MVs from high soluble proteins, we used gel sieving column chromatography method.

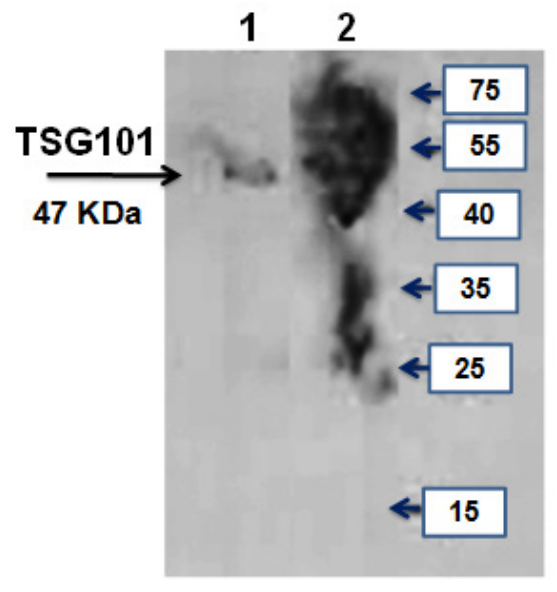

Figure I Western blot image showing urinary micro-vesicles preparations isolated from normal urine (lane-I) and abundant proteins containing urine (lane -2). Proteinuria MVs showed high soluble proteins contamination and problem in getting specific band of TSG IOI, which is vesicle marker. Normal urine MVs showed specific TSGIOI band.

We concentrated MVs using differential centrifugation as described previously..$^{2,7}$ Briefly, 200ml urine sample was centrifuged at $1,500 \mathrm{xg}$ at $25^{\circ} \mathrm{C}$ followed by $17,000 \mathrm{xg}$ for $10 \mathrm{~min}$ at $37^{\circ} \mathrm{C}$. The $17,000 \mathrm{xg}$ supernatant was saved (supernatant 'A') and 17,000xg pellet was re-suspended in isolation solution $(250 \mathrm{mM}$ sucrose, $10 \mathrm{mM}$ triethanolamine $(\mathrm{pH} 7.6)$ containing dithiothreitol (DTT) $(200 \mathrm{mg} /$ $\mathrm{ml}$ ) at $37^{\circ} \mathrm{C}$ for $5-10 \mathrm{~min}$. During incubation, sample was thoroughly mixed using a vortex at an interval of every $2-3 \mathrm{~min}$ till the solution became transparent (approximate time $\sim 15 \mathrm{~min}$ ). Isolation solution was added to the sample to a final volume of $8 \mathrm{ml}$ and centrifuged again at $17,000 \mathrm{xg}$ for $15 \mathrm{~min}$ at $25^{\circ} \mathrm{C}$. The supernatant obtained (supernatant ' $\mathrm{B}$ ') was added to supernatant ' $\mathrm{A}$ ' and named supernatant 
' $C$ '. Supernatant ' $\mathrm{C}$ ' was finally transferred to ultra-centrifuge tubes (Beckman coulter, PA \#344367, USA) and run in an ultracentrifuge (Beckman coulter LE80, USA) at $200,000 \times \mathrm{g}$ for 1 hour at $25^{\circ} \mathrm{C}$ to concentrate MVs pellet. Earlier studies show that Tomm-Horsfall Protein (THP) forms a meshwork around MVs and sediment along with MVs at $17,000 \mathrm{xg}$ centrifugation step. ${ }^{7}$ THP, also known as uromodulin, is a glycoprotein produced by thick ascending limb of the loop of Henle and abundantly secreted to the urine. DTT treatment to $17,000 \mathrm{xg}$ pellet released MVs entrapped into THP meshwork. The released MVs were then sediment following ultracentrifugation. After ultracentrifugation, the supernatant was discarded and MV pellet was suspended in $500 \mu \mathrm{l}$ Tris- $\mathrm{HCl}, \mathrm{pH} 7.4$.

Sephadex G-25 beads were weighed and soaked overnight in $20 \mathrm{mM}$ Tris-Cl, $\mathrm{pH}$ 7.4. A clean glass column was clamped, packed with soaked sephadex G-25 beads and equilibrated with $20 \mathrm{mM}$ Tris- $\mathrm{HCl}, \mathrm{pH}$ 7.4. The bed volume of the column was calculated as $8.0 \mathrm{ml}$. The MVs pellet, suspended in Tris- $\mathrm{HCl}, \mathrm{pH} 7.4$ was applied onto the column and allowed to run with Tris- $\mathrm{HCl}(20 \mathrm{mM}, \mathrm{pH} 7.4)$ buffer at a flow rate of $1 \mathrm{~min} 20 \mathrm{sec}$ per fraction $(1.0 \mathrm{ml}$ per fraction). Optical density at $280 \mathrm{~nm}$ of all fractions were measured, (Figure 2) depicts the line chromatogram of all collected fractions. Three peaks were observed and identified as; peak 1, peak 2, and peak 3. All the fractions were lyophilized and concentrated in suspension solution (1.5\% SDS and 50mM Tris-HCl; pH 6.8). Laemmeli sample buffer (2x) was added to concentrated fractions and thoroughly mixed using a vortex. The fractions were then incubated at $60^{\circ} \mathrm{C}$ for $25 \mathrm{~min}$ and some of the fractions $(5,7,9,11,15$ and 21) were processed for Western blot using TSG101 antibody (Abcam, USA). Western blot analysis showed specific TSG101 band with fractions $11^{\text {th }}, 15^{\text {th }}$ and $21^{\text {st }}$ and contamination was observed infractions 5, 7 and 9. This result represented that after fraction 11 the preparations contain pure urinary vesicles (Figure 3).

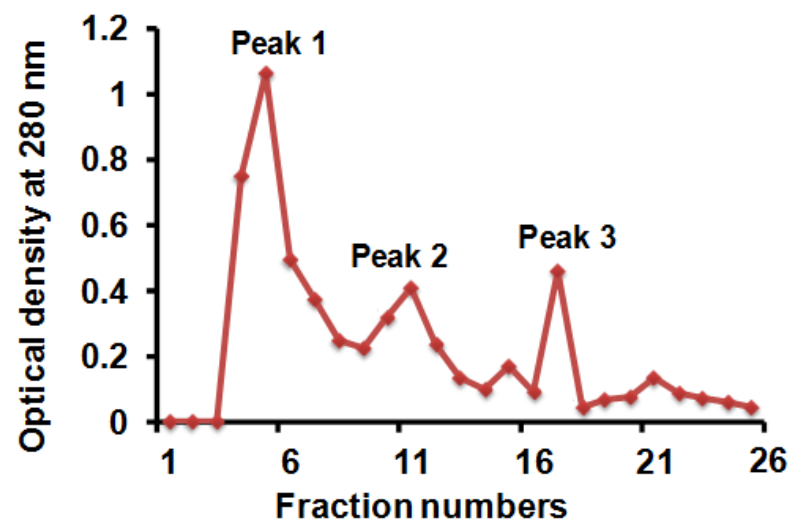

Figure 2 Chromatogram of Sepahdex G-25 column eluted fractions. Three peaks were observed in the chromatogram: peak I, peak 2 and peak 3.

Pooling active fractions; pool I (5-10), and pool II (11-21) allowed us to compare the purification process and determine if other biomarkers could be detected and evaluated. The pooled fractions were analyzed and compared via SDS-PAGE and Western blot processing using apoptosis-linked gene-2 interacting protein [Alix, Abcam USA(another microvesicles marker)] and WT-1[Abcam, USA (proteinuria marker; 2)] antibody. Western blot analysis represented that purified pool II showed the presence of Alix and WT-1 bands. Purified pool I could not produce specific bands of Alix and WT-1 that showed the contamination of soluble proteins in pool I (Figure 4). We have earlier reported the presence of WT-1 in urinary exosomes as nephropathy marker in type -1 diabetic population. ${ }^{2}$ In earlier studies, Rood et al. ${ }^{5}$ used HPLC silica column for the purification of nephrotic urine MVs and obtained MVs-related proteins in nephrotic urine. However, our method is cost effective, does not require sophisticated instrumentation facility and easy to perform. It could be used in routine use for effective isolation of MVs from abundant protein containing urine (protein 4+) and thus useful for biomarker identification.

\section{Fraction numbers}

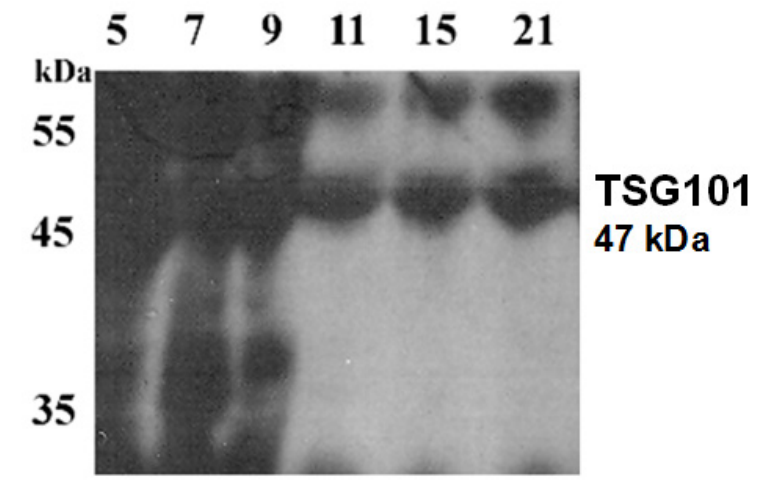

Figure 3 Western blot image of sepadex G-25 eluted column fractions $\left(5^{\text {th }}\right.$, $7^{\text {th }}, 9^{\text {th }}, \mathrm{II}^{\text {th }}, 15^{\text {th }}$ and $\left.2 \mathrm{I}^{\text {st }}\right)$ using anti-TSG IOI antibody. Fraction $\mathrm{II}^{\text {th }}, 15^{\text {th }}$ and $2 I^{\text {st }}$ showed specific band of TSGIOI.

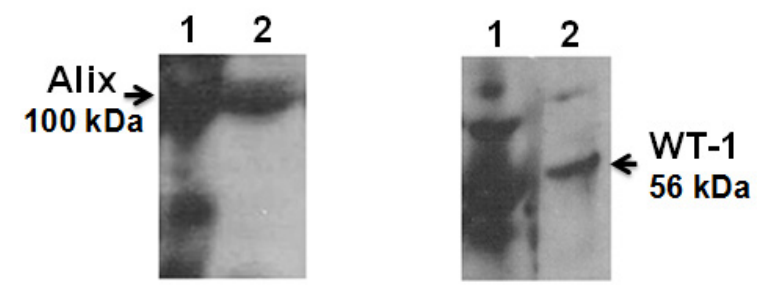

Figure 4 Western blot images of two pools (pool I and Pool II) using anti-Alix and Wilms' tumor (WT-I) antibody. Pool II showed specific presence of two markers as compared to pool I that showed contamination of soluble proteins. Here, lane I, Pool I and lane 2, pool II.

\section{Limitation of the study}

i. MVs content may get lose in the column purification process. We are not sure about their quantity as MVs content is different in different individuals and also vary in different disease state conditions. However, for an efficient and effective Western blot analysis only $40-80 \mu \mathrm{g} \mathrm{MVs} / \mathrm{lane}$ is required and our purification process generates more than required amount of MVs quantity. Despite that, running again the un-purified pool fraction (pool I) through column chromatography can generate more quantity of MVs for further analysis.

ii. Purification process generated MVs that presented extra band with TSG101 specific band by Western blot analysis. We are not sure about the identity of other bands but we confirmed the major band at $47 \mathrm{kDa}$ that localized at correct size and started from fraction $11^{\text {th }}$ with anti-TSG101 antibody (Figure 3). The partially purified pool fractions (fraction 11 onwards) were also found effective with exosomes specific another marker Alix and proteinuria specific WT-1.2 These markers further validated the purity of the MVs and importance of this partially purified fraction to be further used for biomarker identification. 


\section{Acknowledgements}

This work was supported by SGPGI- Intramural research grant to ST and Indian Council of Medical Research associate fellowship to AK (3/1/3/5/2011-RHN).

\section{Conflict of interest}

The author declares no conflict of interest

\section{References}

1. Salih M, Zietse R, Hoorn EJ. Urinary extracellular vesicles and the kidney: biomarkers and beyond. Am J Physiol Renal Physiol. 2014;306(11):F1251-F1259.

2. Kalani A, Mohan A, Godbole MM, et al. Wilm's tumor-1 protein levels in urinary exosomes from diabetic patients with or without proteinuria PLoS One. 2013;8(3):e60177.
3. Zhou H, Yuen PS, Pisitkun T, et al. Collection, storage, preservation, and normalization of human urinary exosomes for biomarker discovery. Kidney Int. 2006;69(8):1471-1476.

4. Merchant ML, Powell DW, Wilkey DW, et al. Microfiltration isolation of human urinary exosomes for characterization by MS. Proteomics Clin Appl. 2010;4(1):84-96.

5. Rood IM, Deegens JK, Merchant ML, et al. Comparison of three methods for isolation of urinary microvesicles to identify biomarkers of nephrotic syndrome. Kidney Int. 2010;78(8):810-816.

6. Akao Y, Iio A, Itoh T, et al. Microvesicle-mediated RNA molecule delivery system using monocytes/macrophages. Mol Ther. 2011;19(2):395-399.

7. Fernandez-Llama P, Khositseth S, Gonzales PA, et al. Tamm-Horsfall protein and urinary exosome isolation. Kidney Int. 2010;77(8):736-742. 